\section{BRAZIULIAN JOURNAL}

OF MEDICAL AND BIOLOGICAL RESFARCH

www.bjournal.com.br
ISSN 0100-879X

Volume 44 (7) 606-728 July 2011

BIOMEDICAL SCIENCES

AND

CLINICAL INVESTIGATION

Braz J Med Biol Res, July 2011, Volume 44(7) 720-724

doi: 10.1590/S0100-879X2011007500079

Air pollution and cardiovascular hospital admissions in a medium-sized city in São Paulo State, Brazil

L.F.C. Nascimento

The Brazilian Journal of Medical and Biological Research is partially financed by

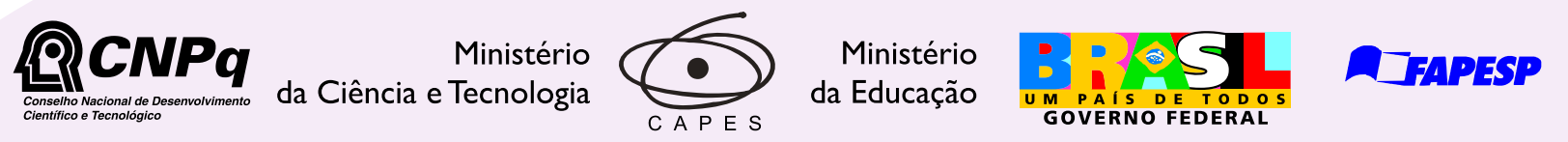

Institutional Sponsors

sup?o
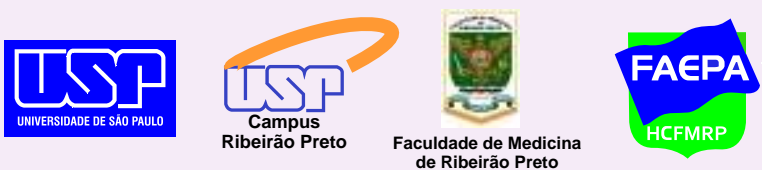

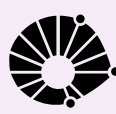

$\oplus$ SHIMADZU

GE Healthcare
Hotsite of proteomics metabolomics developped by:

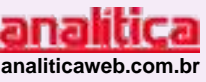

Thermo SCIENTIFIC 


\title{
Air pollution and cardiovascular hospital admissions in a medium-sized city in São Paulo State, Brazil
}

\author{
L.F.C. Nascimento
}

Departamento de Medicina and Programa de Pós-Graduação em Ciências Ambientais, Universidade de Taubaté, Taubaté, SP, Brasil

\begin{abstract}
The objective of the present study was to estimate the contribution of environmental pollutants to hospital admissions for cardiovascular disease. A time series ecological study was conducted on subjects aged over 60 years and living in São José dos Campos, Brazil, with a population near 700,000 inhabitants. Hospital admission data of public health patients (SUS) were obtained from DATASUS for the period between January 1, 2004 and December 31, 2006, according to the ICD-10 diagnoses $\mathrm{I} 20$ to I22 and I24. Particulate matter with less than $10 \mu \mathrm{m}$ in aerodynamic diameter, sulfur dioxide and ozone were the pollutants examined, and the control variables were mean temperature and relative humidity. Data on pollutants were obtained from the São Paulo State Sanitary Agency. The generalized linear model Poisson regression with lags of up to 5 days was used. There were 1303 hospital admissions during the period. Exposure to particulate matter was significantly associated with hospitalization for cardiovascular disease 3 days after exposure $(R R=1.006 ; 95 \% \mathrm{Cl}=1.000$ to 1.010$)$ and an increase of $16 \mu \mathrm{g} / \mathrm{m}^{3} \mathrm{was}$ associated with a $10 \%$ increase in risk of hospitalization; other pollutants were not associated with hospitalization. Thus, it was possible to identify the role of exposure to particulate matter as an environmental pollutant in hospitalization for cardiovascular disease in a medium-sized city in Southeastern Brazil.
\end{abstract}

Key words: Air pollution; Particulate matter; Cardiovascular diseases; Time series studies

\section{Introduction}

The incidence and prevalence of cardiovascular diseases have increased over the last decades and have become one of the main causes of death among adults $(1,2)$. In metropolitan areas, the increase in the level of atmospheric pollutants becomes an additional risk factor for such diseases $(3,4)$. Many epidemiological studies for the quantification of the effects of atmospheric pollution on human health have reported mortality as a result, with a strong association between the result and acute and chronic exposure $(5,6)$.

Interest in investigating morbidity for cardiovascular diseases has increased in recent years. The association between air pollution and emergency room visits for ischemic cardiovascular disease has been demonstrated recently in São Paulo, Brazil. The concentration of carbon monoxide (CO) in air has been associated not only with angina but also with acute myocardial infarction after a few days of exposure depending on personal characteristics (7). In another study, also carried out in the vicinity of São Paulo, the pollutants studied were associated with admission due to myocardial infarction, with emphasis on sulfur dioxide (8). Other effects on the cardiovascular system such as arterial hypertension and alteration in the heart rate variability have also been observed in São Paulo. The relationship between cardiovascular diseases and air pollution has been little explored, and the physiopathological bases of this association are still unexplained. Studies have suggested two possible mechanisms explaining cardiovascular damage caused by the action of pollutants. The first involves changes in the modulation of the autonomic nervous system, as indicated by the observation of a decrease in heart rate variability in relation to the level of pollutants. Some diseases of the cardiovascular system, such as acute myocardial infarction and sudden death, are associated with alterations in the autonomic nervous system. Another possible mechanism is related to the increase in fibrinogen and circulating

Correspondence: L.F.C. Nascimento, Rua Durval Rocha, 500, 12515-710 Guaratinguetá, SP, Brasil. Fax: +55-12-3123-2835

E-mail: luiz.nascimento@unitau.br

Received November 21, 2010. Accepted June 3, 2011. Available online June 24, 2011. Published July 25, 2011. 
inflammation factors, which lead to an increase in blood viscosity and favor coagulation (9-11). In 2007, 60 thousand admissions for myocardial infarction and angina occurred in São Paulo, reaching a cost of R\$150 million, and 3400 admissions occurred in the Paraíba Valley, reaching a cost of a little less than $\mathrm{R} \$ 9$ million (in 2011 , US $\$ 1 \approx \mathrm{R} \$ 1.70$ ) (12). It is generally agreed that the fleet of vehicles is one, if not the main source generating sulfur dioxide pollutants, particulate matter and ozone. This being so, we can understand the impact of such pollutants on the admissions for cardiovascular diseases in large cities. Nevertheless, are the effects of pollutants on the emergence of cardiovascular diseases similar to those in a medium-sized town?

The objective of the present study was to estimate these effects on admissions for ischemic heart disease (IHD) such as acute infarction and angina in São José dos Campos, São Paulo, a medium-sized city.

\section{Material and Methods}

This was an ecological time-series study using secondary admission data for IHD (ICD-10 diagnoses: I20 to I22 and 124), obtained from the Information System of the National Health Care System (DATASUS) for the period from January 1, 2004 to December 31, 2006, including subjects of both genders aged 60 years or more. The study was conducted in the city of São José dos Campos, a city in the Paraíba Valley, State of São Paulo, located $91 \mathrm{~km}$ from São Paulo city. Its population is approximately 700,000 inhabitants. São José dos Campos is an industrial, shopping and service center serving a population of approximately 2 million inhabitants from the Paraíba Valley and Minas Gerais State, located at $23^{\circ} 11^{\prime}$ latitude South and $45^{\circ} 53^{\prime}$ longitude West. The São Paulo State Sanitary Agency (CETESB) provided data for the estimated pollutants in São José dos Campos, where a monitoring station is located. Pollutants estimated by this station are sulfur dioxide $\left(\mathrm{SO}_{2}\right)$, particulate matter with less than $10 \mu \mathrm{m}$ of aerodynamic diameter $\left(\mathrm{PM}_{10}\right)$, and ozone $\left(\mathrm{O}_{3}\right)$. Mean hourly values of measurements made from 1:00 am to 12:00 pm were considered. Meteorological data, air relative humidity, daily minimum and median temperatures were obtained from the Foundation for Science, Technology and Space Applications (FUNCATE) (13).

\section{Statistical analysis}

The description of each variable in terms of valid number of mean, median, minimum and maximum observation values, standard deviation, and interquartile differences was the first step taken. The daily number of admissions for IHD was considered as a dependent variable and the $\mathrm{PM}_{10}, \mathrm{SO}_{2}$ and $\mathrm{O}_{3}$ pollutant concentrations quantified as $\mu \mathrm{g} / \mathrm{m}^{3}$ were considered as independent variables controlled for climatic variables such as air relative humidity and daily median temperature.

The generalized linear Poisson regression model was used for counting purposes. The number of degrees of freedom for the spline was chosen to minimize autocorrelations among residues. As the effect of atmospheric pollution on IHD morbidity may be distributed along the time, the number of events on a single day may be due to the exposure to air pollution not only on this day but along the previous day, with no consensus in the literature about the lag days. Therefore, in order to better investigate this pattern, we included in single pollutant models their daily levels ranging from the current day to 5 days before the visit to the emergency room. The risks of admission and the percent increase (INC) of these risks were estimated according to the increase observed in the interquartile difference (IQD) of each pollutant, according to the expression INC = (RR - 1) x 100, where RR (relative risk) is defined as $\exp$ (coeff $x$ IQD), where coeff is the pollutant coefficient obtained by the Poisson regression.

\section{Results}

During the 1096 days of the study, 1303 admissions were registered, with ICD-10 diagnoses of I20 to I22 and/ or 124. The mean, minimum and maximum values, the respective standard deviations, and IQD are presented in Table 1. The distribution of the daily levels of pollutants, in $\mu \mathrm{g} / \mathrm{m}^{3}$, are shown in Figure 1.

Complete information of all daily hospital admissions was available; missing temperature and humidity data were restricted to the interval from January 1 to February 18, 2004, when the measurement of these data was initiated by FUNCATE. The missing data for pollutants were randomly spread along the days of the study period. Table 2 shows the Pearson correlation coefficients, which revealed a high correlation between pollutants and meteorological variables $(P<0.01)$ except between $\mathrm{PM}_{10}$ and mean temperature. Table 3 presents the coefficients obtained with the general linear model, with the respective standard errors of the mean. Only exposure to particulate matter with a lag of 3

Table 1. Descriptive analysis of the variables included in the Poisson regression model.

\begin{tabular}{lcccc}
\hline & Mean $\pm \mathrm{SD}$ & Maximum & Minimum & IQD \\
\hline Hospital admission & $1.19 \pm 1.21$ & 9 & 0 & 2 \\
Relative humidity $(\%)$ & $80.1 \pm 6.5$ & 99.3 & 54 & 8 \\
Mean temperature $\left({ }^{\circ} \mathrm{C}\right)$ & $20.2 \pm 2.4$ & 27.9 & 13.1 & 4 \\
$\mathrm{SO}_{2}\left(\mu \mathrm{g} / \mathrm{m}^{3}\right)$ & $4.3 \pm 3.1$ & 31 & 1 & 3 \\
$\mathrm{PM}_{10}\left(\mu \mathrm{g} / \mathrm{m}^{3}\right)$ & $25.5 \pm 13.7$ & 100 & 6 & 16 \\
$\mathrm{O}_{3}\left(\mu \mathrm{g} / \mathrm{m}^{3}\right)$ & $75.4 \pm 31.5$ & 232 & 20 & 40 \\
\hline
\end{tabular}

$\mathrm{IQD}=$ interquartile difference; $\mathrm{SO}_{2}=$ sulfur dioxide; $\mathrm{PM}_{10}=$ particulate matter with less than $10 \mu \mathrm{m}$ in aerodynamic diameter; $\mathrm{O}_{3}=$ ozone. 
Table 2. Pearson correlation analysis of air pollutants and weather variables during the period analyzed.

\begin{tabular}{lccllll}
\hline & $\begin{array}{c}\text { Relative } \\
\text { humidity }\end{array}$ & $\begin{array}{c}\text { Mean } \\
\text { temperature }\end{array}$ & $\mathrm{SO}_{2}$ & $\mathrm{PM}_{10}$ & $\mathrm{O}_{3}$ \\
\hline Relative humidity (\%) & 1 & & & & & \\
Mean temperature $\left({ }^{\circ} \mathrm{C}\right)$ & -0.22 & 1 & & & \\
$\mathrm{SO}_{2}\left(\mu \mathrm{g} / \mathrm{m}^{3}\right)$ & -0.18 & -0.23 & 1 & & \\
$\mathrm{PM}_{10}\left(\mu \mathrm{g} / \mathrm{m}^{3}\right)$ & -0.35 & -0.12 & $0.49^{*}$ & 1 & \\
$\mathrm{O}_{3}\left(\mu \mathrm{g} / \mathrm{m}^{3}\right)$ & -0.46 & 0.37 & 0.27 & 0.38 & 1 \\
\hline
\end{tabular}

$\mathrm{SO}_{2}=$ sulfur dioxide; $\mathrm{PM}_{10}=$ particulate matter with less than 10 $\mu \mathrm{m}$ in aerodynamic diameter; $\mathrm{O}_{3}=$ ozone. ${ }^{*} \mathrm{P}>0.05$.

Table 3. Regression coefficients and $95 \%$ confidence interval $(95 \% \mathrm{Cl})$ according to lag structure for hospital admission due to cardiovascular disease, São José dos Campos, 2004-2006.

\begin{tabular}{lrc}
\hline & Coefficient & $95 \% \mathrm{Cl}$ \\
\hline $\mathrm{SO}_{2}$ & & \\
Concurrent day & 0.00818 & $-0.01459 ; 0.03095$ \\
LAG 1 & -0.01589 & $-0.04108 ; 0.00931$ \\
LAG 2 & 0.00273 & $-0.02069 ; 0.02616$ \\
LAG 3 & -0.01605 & $-0.04063 ; 0.00854$ \\
LAG 4 & 0.00227 & $-0.02064 ; 0.02518$ \\
LAG 5 & 0.01362 & $-0.00819 ; 0.03544$ \\
PM 10 & & \\
Concurrent day & -0.00232 & $-0.00757 ; 0.00294$ \\
LAG 1 & -0.00099 & $-0.00634 ; 0.00436$ \\
LAG 2 & -0.00194 & $-0.00724 ; 0.00336$ \\
LAG 3 & $\mathbf{0 . 0 0 5 7 8}$ & $\mathbf{0 . 0 0 0 2 3} ; 0.01033$ \\
LAG 4 & 0.00093 & $-0.00419 ; 0.00605$ \\
LAG 5 & 0.00149 & $-0.00355 ; 0.00653$ \\
O $_{3}$ & & \\
Concurrent day & -0.00011 & $-0.00251 ; 0.00229$ \\
LAG 1 & 0.00119 & $-0.00012 ; 0.00361$ \\
LAG 2 & -0.00042 & $-0.00285 ; 0.00201$ \\
LAG 3 & 0.00009 & $-0.00232 ; 0.00249$ \\
LAG 4 & 0.00081 & $-0.00156 ; 0.00319$ \\
LAG 5 & -0.00115 & $-0.00355 ; 0.00126$ \\
\hline
\end{tabular}

The value in bold indicates significant association between air pollutant and outcome. $\mathrm{SO}_{2}=$ sulfur dioxide; $\mathrm{PM}_{10}=$ particulate matter with less than $10 \mu \mathrm{m}$ in aerodynamic diameter; $\mathrm{O}_{3}$ = ozone.

days was associated with hospitalization for cardiovascular disease.

The percent increases in RR for admissions were not high, except for $\mathrm{PM}_{10} 3$ days after exposure, which reached up to $10 \%$.

\section{Discussion}

This was the first study on the effect of air pollutants on

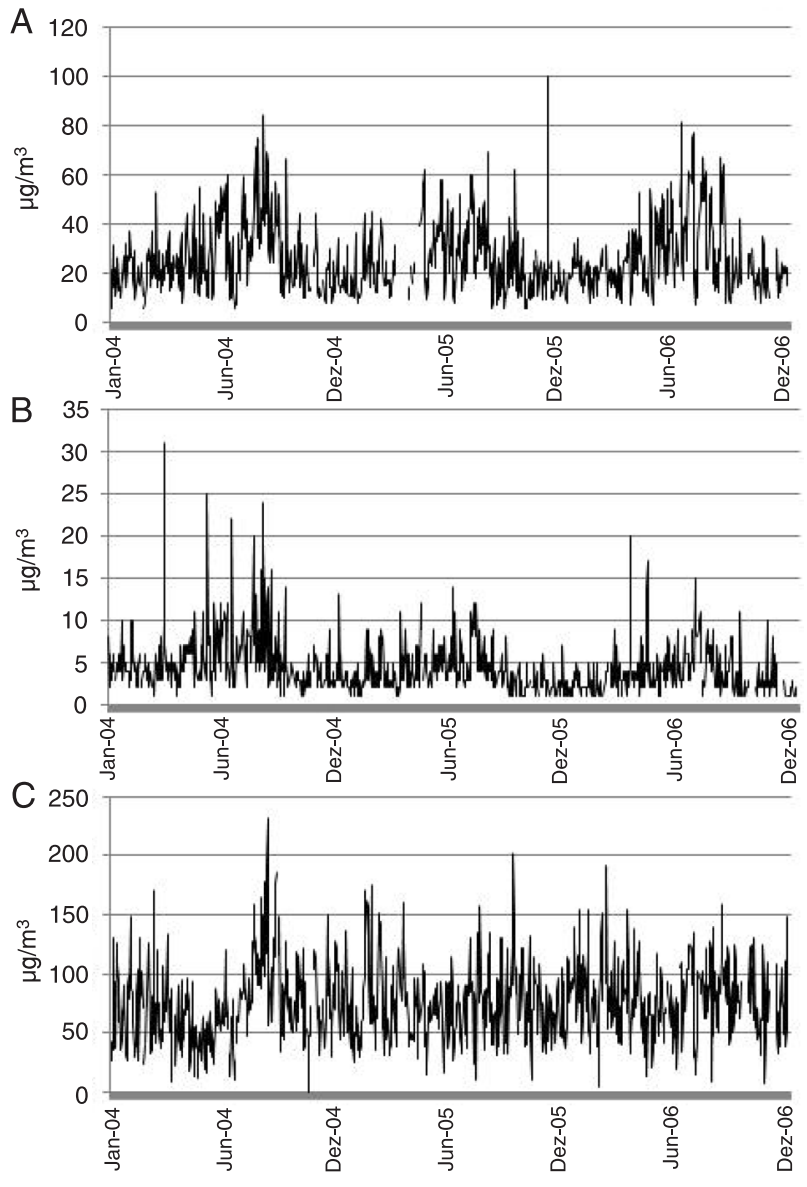

Figure 1. Daily values of pollutant particulate matter $(A)$, sulfur dioxide (B), and ozone (C) during the study period, São José dos Campos, Brazil, 2004-2006. (Source: São Paulo State Sanitary Agency).

admissions for IHD carried out outside large cities. Exposure to particulate matter has been identified as a factor associated with admissions for IHD in São José dos Campos, a medium-sized city located in the Paraíba Valley. The estimated risk identified in this study regarding particulate matter was of small magnitude $(R R=1.006)$ and the $R R$ increased 1.096 when considering an increase of $16 \mu \mathrm{g} /$ $\mathrm{m}^{3}$ in the concentration of this pollutant.

The association of exposure to particulate matter with admissions for IHD was shown by Gouveia et al. (14) in a 4-year time series study of admission data for people over 65 years of age at hospitals in the municipality of São Paulo. The risk of admission was 1.010 for cardiovascular disease and 1.15 for ischemic disease when there was an increase of $10 \mu \mathrm{g} / \mathrm{m}^{3}$ in the concentration of this pollutant. These investigators did not study the effects during lags. It is important to point out that the mean concentrations of $\mathrm{PM}_{10}$ in the cited study were $54.5 \mu \mathrm{g} / \mathrm{m}^{3}$, practically twice as high as those found in our study. 
The present findings indicate that the 3rd day after exposure was a risk for hospital admission. These data are not consistent with those reported by Cendon et al. (8), which indicate that the same day of exposure (lag 0) was that associated with hospital admission for myocardial infarction. The mean value of particulate matter detected by these investigators was $48.3 \mu \mathrm{g} / \mathrm{m}^{3}$, and for each increase of 22 $\mu \mathrm{g} / \mathrm{m}^{3}$, the risk of admission observed was a little less than $5 \%$. The cited study was carried out at 112 hospitals linked to the Unified Health System (SUS), in the municipality of São Paulo in 1998 and 1999.

In another study, also carried out in the municipality of São Paulo (14), $\mathrm{PM}_{10}$ was associated with admissions for cardiovascular diseases. The cited study did not use models including all pollutants of model with combinations of pollutants, in contrast to our study, which used modeling with three pollutants simultaneously, with adjustments for humidity and mean temperature.

Pereira-Filho et al. (15) did not find any association between exposure to particulate matter and admission for cardiovascular diseases, including arterial hypertension in their series of subjects aged 18 years or more, with or without diabetes as co-morbidity. These investigators used lags of up to 2 days.

The present study did not detect any association between exposure to $\mathrm{SO}_{2}$ and admissions, in contrast to other studies conducted in São Paulo $(9,14-16)$. A possible explanation may be related to the high $\mathrm{SO}_{2}$ values of 13 to $17 \mu \mathrm{g} / \mathrm{m}^{3}$ detected in the cited studies, as opposed to the value of $4.3 \mu \mathrm{g} / \mathrm{m}^{3}$ detected in São José dos Campos in the present study.

Exposure to ozone was not associated with admissions for IHD, in agreement with data obtained in studies conducted in São Paulo (14-16), but not with data reported by Cendon et al. (8) who observed an acute effect of ozone at concentrations similar to those detected in the present study. These investigators studied subjects older than 64 years using a model that considered one pollutant at a time, in contrast to the present study. In these studies, the temperature was reported as the mean temperature, unlike other studies $(8,9)$.

The use of the generalized linear model, instead of the generalized additive model $(6,7,14,16)$, is justified by the fact that there is no substantial difference between their results (17).

Despite the small magnitude of the RR found here $(R R=1.006)$, the impact of air pollution on the health of the population must be substantial considering the large number of individuals exposed to it. The results represent a quantitative approximation of the impact of atmospheric pollution on the health of the population. It is important to point out that the factor studied here, i.e., hospitalization, is a serious problem but it is only one of the negative effects caused by air pollution.

Other less serious effects such as the occurrence of symptoms, use of medication and reduction of physical activity, among others, were not assessed in the present study. Even though these items are considered to be less serious for an individual's health, they are of great importance to public health due to their very frequent occurrence and also to their negative impact on the quality of life of the population, with negative economic consequences.

This study was based on secondary data obtained from an official, stable and reliable source and widely used in technical and scientific work (18). It is worth noting that these records concern hospitalizations occurring in the public health network, which does not include care provided by health insurance. Thus, the present results reflect the effects of air pollution on the population that uses public services, which represents, in fact, most of the Brazilian population (around 85\%).

On the other hand, the information available in the Hospital Information System of SUS, used in the present study, is produced for accounting purposes and not only for epidemiological studies, and may therefore have a certain degree of inaccuracy. There may still have been a certain degree of underestimation of the frequency of certain grievances, considering the technological profile of the assistance network, apart from the mistakes in the codification of diagnoses. Nowadays, diagnostic confirmation of hospital admission is done a posteriori, with the purpose of reducing diagnostic errors on the occasion of hospital admission.

In ecological studies of temporal series carried out on a daily basis, factors whose distribution does not vary daily do not act as possible confounding variables. Therefore, the quality of information about hospital admissions and other possible problems of these data bases, which do not vary daily and which are not correlated with the levels of air pollution, have little implications for the estimates of the effect found.

On the other hand, it is worth noting that this was an ecological study in which no individual information about exposure to disease was available. It is not known, for instance, whether the hospitalized individuals were actually more exposed to reported levels of air pollution. It can be assumed that exposure measured daily was homogeneous throughout the city, and that individuals were similarly exposed, otherwise the problem would be unsolved.

Estimating the risk for the population health with regard to air pollution is the first step in planning and implementing actions aiming at a healthier environment. Production of technically based data is fundamental for the formulations of public policies and decision-making that promote socio-economic development apart from considering environmental issues and people's life quality.

Thus, it was possible to determine that exposure to particulate matter with less than $10 \mu \mathrm{m}$ in aerodynamic diameter was a risk factor for cardiovascular disease on the 3rd day after exposure, in São José dos Campos, Brazil. 


\section{References}

1. Brunekreef $\mathrm{B}$, Holgate ST. Air pollution and health. Lancet 2002; 360: 1233-1242.

2. Pope CA III, Burnett RT, Thun MJ, Calle EE, Krewski D, Ito K, et al. Lung cancer, cardiopulmonary mortality, and long-term exposure to fine particulate air pollution. JAMA 2002; 287: 1132-1141.

3. Sunyer J, Ballester F, Tertre AL, Atkinson R, Ayres JG, Forastiere $\mathrm{F}$, et al. The association of daily sulfur dioxide air pollution levels with hospital admissions for cardiovascular diseases in Europe (The Aphea-II study). Eur Heart J 2003; 24: $752-760$.

4. Zanobetti A, Schwartz J, Samoli E, Gryparis A, Touloumi G, Peacock $\mathrm{J}$, et al. The temporal pattern of respiratory and heart disease mortality in response to air pollution. Environ Health Perspect 2003; 111: 1188-1193.

5. Schwartz J. Harvesting and long term exposure effects in the relation between air pollution and mortality. Am J Epidemiol 2000; 151: 440-448.

6. Braga AL, Zanobetti A, Schwartz J. The lag structure between particulate air pollution and respiratory and cardiovascular deaths in 10 US cities. J Occup Environ Med 2001; 43: 927-933.

7. Lin CA, Amador Pereira LA, de Souza Conceicao GM, Kishi HS, Milani R Jr, Ferreira Braga AL, et al. Association between air pollution and ischemic cardiovascular emergency room visits. Environ Res 2003; 92: 57-63.

8. Cendon S, Pereira LA, Braga AL, Conceicao GM, Cury JA, Romaldini $\mathrm{H}$, et al. Air pollution effects on myocardial infarction. Rev Saude Publica 2006; 40: 414-419.

9. de Paula Santos U, Braga AL, Giorgi DM, Pereira LA, Grupi CJ, Lin CA, et al. Effects of air pollution on blood pressure and heart rate variability: a panel study of vehicular traffic controllers in the city of São Paulo, Brazil. Eur Heart J 2005; 26: $193-200$
10. Holguin F, Tellez-Rojo MM, Hernandez M, Cortez M, Chow JC, Watson JG, et al. Air pollution and heart rate variability among the elderly in Mexico City. Epidemiology 2003; 14: 521-527.

11. Metzger KB, Tolbert PE, Klein M, Peel JL, Flanders WD, Todd $\mathrm{K}$, et al. Ambient air pollution and cardiovascular emergency department visits. Epidemiology 2004; 15: 46-56.

12. Ministério da Saúde. Secretaria Executiva. DATASUS Informações de Saúde. Morbidade e informações epidemiológicas. http://tabnet.datasus.gov.br/cgi/tabcgi.exe?sih/ cnv/mrsp.def. Accessed May 10, 2010.

13. FUNCATE. Fundação de Ciência, Aplicações e Tecnologia Espaciais. http://strademaweb.funcate.org.br/STRADEMAWEB/. Accessed May 10, 2010.

14. Gouveia N, de Freitas CU, Martins LC, Marcilio IO. [Respiratory and cardiovascular hospitalizations associated with air pollution in the city of São Paulo, Brazil]. Cad Saude Publica 2006; 22: 2669-2677.

15. Pereira Filho MA, Pereira LA, Arbex FF, Arbex M, Conceicao GM, Santos UP, et al. Effect of air pollution on diabetes and cardiovascular diseases in São Paulo, Brazil. Braz J Med Biol Res 2008; 41: 526-532.

16. Martins LC, Pereira LA, Lin CA, Santos UP, Prioli G, Luiz OC, et al. The effects of air pollution on cardiovascular diseases: lag structures. Rev Saude Publica 2006; 40: 677-683.

17. Conceição GMS, Saldiva PHN, Singer JM. Modelos MLG e MAG para análise da associação entre poluição atmosférica e marcadores de morbi-mortalidade: uma introdução baseada em dados da cidade de São Paulo. Rev Bras Epidemiol 2001; 4: 206-219.

18. Veras CM, Martins MS. [Reliability of data from Authorization Forms for Hospital Admittance, Rio de Janeiro, Brazil]. Cad Saude Publica 1994; 10: 339-355. 